\title{
Quantum memory for entangled continuous-variable states
}

\author{
K. Jensen ${ }^{1 \dagger}$, W. Wasilewski ${ }^{1 \dagger t}$, H. Krauter ${ }^{1}$, T. Fernholz ${ }^{1 *}$, B. M. Nielsen ${ }^{1}$, M. Owari ${ }^{2}$, M. B. Plenio ${ }^{2}$, \\ A. Serafini ${ }^{3}$, M. M. Wolf ${ }^{1}$ and E. S. Polzik ${ }^{1 \star}$
}

\begin{abstract}
A quantum memory for light is a key element for the realization of future quantum information networks ${ }^{1-3}$. Requirements for a good quantum memory are versatility (allowing a wide range of inputs) and preservation of quantum information in a way unattainable with any classical memory device. Here we demonstrate such a quantum memory for continuousvariable entangled states, which play a fundamental role in quantum information processing ${ }^{4-6}$. We store an extensive alphabet of two-mode $6.0 \mathrm{~dB}$ squeezed states obtained by varying the orientation of squeezing and the displacement of the states. The two components of the entangled state are stored in two room-temperature cells separated by $0.5 \mathrm{~m}$, one for each mode, with a memory time of $1 \mathrm{~ms}$. The true quantum character of the memory is rigorously proved by showing that the experimental memory fidelity $0.52 \pm 0.02$ significantly exceeds the benchmark of 0.45 for the best possible classical memory for a range of displacements.
\end{abstract}

The continuous-variable regime represents one of the principal avenues towards the realization of quantum information processing and communication ${ }^{4-6}$. In the optical domain it operates with well-known optical modulation and detection techniques and allows for deterministic quantum operations. In the atomic domain it has been developed on the platform of atomic ensembles ${ }^{2,3,7}$. Advances in the realization of continuous-variable quantum protocols include unconditional quantum teleportation involving light $^{8}$ and atoms ${ }^{9}$, a number of results on memory ${ }^{2,3,10,11}$ and quantum key distribution ${ }^{12}$. Hybrid continuous/discrete-variable operations ${ }^{13-15}$ paving the road towards continuous-variable quantum computation ${ }^{16,17}$ have also been reported. However, the ability to store non-classical continuous-variable states of light is crucial to enable further progress, in particular, for continuous-variable linear optics quantum computing with offline resources ${ }^{17}$, continuous-variable quantum repeaters ${ }^{18,19}$, entanglement-enhanced quantum metrology, iterative continuousvariable entanglement distillation ${ }^{20}$, continuous-variable clusterstate quantum computation ${ }^{21}$, communication/cryptography protocols involving several rounds ${ }^{22}$ and quantum illumination ${ }^{23}$. Compared with a number of impressive results reporting discrete-variable quantum memories at the single-photon level (see reviews ${ }^{1-3}$ and references therein), there have been very few experiments towards quantum memory for continuousvariable non-classical states. A fractional, $20 \mathrm{nsec}$, delay of $50 \mathrm{nsec}$ pulsed continuous-variable entangled states in the atomic vapour has recently been demonstrated ${ }^{24}$. Memory based on electromagnetically induced transparency for a squeezed vacuum state has been reported ${ }^{25,26}$, albeit with the fidelity below the classical benchmark ${ }^{27}$. Very recently, classical benchmarks for storing displaced squeezed states $^{28,29}$ have been derived, which made experimental implementation of such storage a timely challenge. Such states form an alphabet for continuousvariable quantum information encoding ${ }^{16}$. An exciting feature of displaced squeezed states is that the ratio of the quantum to classical fidelity grows inversely proportionally to the degree of squeezing.

Here we report the realization of a quantum memory for a set of displaced two-mode squeezed states with an unconditionally high fidelity that exceeds the classical benchmark calculated on the basis of the method in ref. 28. The fidelity between the input state and the memory state is a sufficient condition for a memory or a teleportation protocol to be genuinely quantum. In fact, because continuous-variable protocols are deterministic, that is they have a unity efficiency, the fidelity becomes the preferred performance criterion. The experimental fidelity demonstrated here, which exceeds the classical benchmark fidelity, implies that our quantum memory cannot be mimicked by any classical device.

We store a displaced entangled state of two sideband modes of light $\hat{a}_{+}$and $\hat{a}_{-}$with the frequencies $\omega_{ \pm}=\omega_{0} \pm \omega_{\mathrm{L}}$, where $\omega_{0}$ is the carrier frequency of light. The entanglement condition for this Einstein-Podolsky-Rosen state ${ }^{6}$ is $\operatorname{Var}\left(\hat{X}_{+}+\hat{X}_{-}\right)+\operatorname{Var}\left(\hat{P}_{+}-\right.$ $\left.\hat{P}_{-}\right)<2$ (ref. 30) where canonical quadrature operators obey $\left[\hat{X}_{ \pm}, \hat{P}_{ \pm}\right]=i$. For a vacuum state $\operatorname{Var}\left(\hat{X}_{\mathrm{vac}}\right)=\operatorname{Var}\left(\hat{P}_{\mathrm{vac}}\right)=1 / 2$. The entanglement of the $\hat{a}_{+}$and $\hat{a}_{-}$modes is equivalent to simultaneous squeezing of the $\cos \left(\omega_{\mathrm{L}} t\right)$ mode $\hat{x}_{\mathrm{Lc}}=\left(\hat{X}_{+}+\hat{X}_{-}\right) / \sqrt{2}$; $\hat{p}_{\mathrm{Lc}}=\left(\hat{P}_{+}+\hat{P}_{-}\right) / \sqrt{2}$ and the corresponding $\sin \left(\omega_{\mathrm{L}} t\right)$ mode. Before the input state of light undergoes various losses it is a $6 \mathrm{~dB}$ squeezed state. In the photon-number representation for the two modes, the state is $|\Psi\rangle=0.8|0\rangle_{+}|0\rangle_{-}+0.48|1\rangle_{+}|1\rangle_{-}+$ $0.29|2\rangle_{+}|2\rangle_{-}+0.18|3\rangle_{+}|3\rangle_{-}+\cdots$. The displaced squeezed states are produced (Fig. 1a) using an optical parametric amplifier ${ }^{31}$ (OPA) with the bandwidth of $8.3 \mathrm{MHz}$ and two electro-optical modulators (EOMs; see the Methods section for details). The alphabet of quantum states $\Psi_{\mathrm{i}}$, which we refer to as 'initial pure states' (see the inset in Fig. 2 and Table 1) is generated by displacing the two-mode squeezed vacuum state by varying values $\left[\left\langle x_{\mathrm{L}}\right\rangle ;\left\langle p_{\mathrm{L}}\right\rangle\right]=[0,3.8,7.6 ; 0,3.8,7.6]$

\footnotetext{
${ }^{1}$ Niels Bohr Institute, Danish Quantum Optics Center QUANTOP, Copenhagen University, Blegdamsvej 17, 2100 Copenhagen, Denmark, ${ }^{2}$ Institut für Theoretische Physik, Universität Ulm, Albert-Einstein Allee 11, D-89069 Ulm, Germany, ${ }^{3}$ University College London, Department of Physics and Astronomy, Gower Street, London WC1E 6BT, UK. †These authors contributed equally to this work. *Present addresses: Institute of Experimental Physics, Warsaw University, Hoza 69, 00-681 Warszawa, Poland (W.W.); School of Physics and Astronomy, The University of Nottingham, University Park, Nottingham NG7 2RD, UK (T.F.). ${ }^{\star}$ e-mail: polzik@nbi.dk.
} 
a

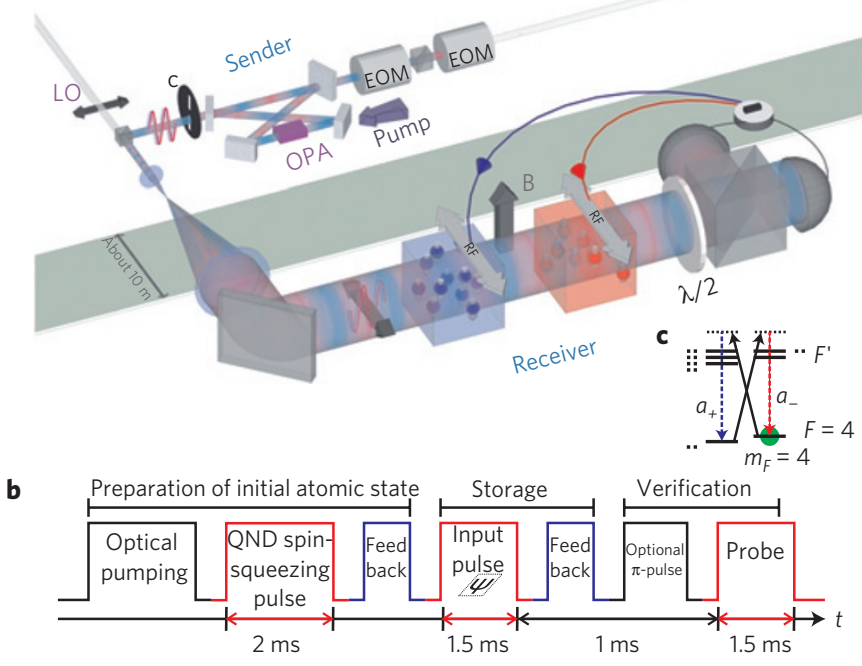

Figure 1 | Set-up and pulse sequence. a, At the sender station two-mode entangled (squeezed) light is generated by the OPA. A variable displacement of the state is achieved by injecting a coherent input into the OPA modulated by EOMs. The output of the OPA is shaped by a chopper, and combined on a polarizing beam splitter with the local oscillator (LO) beam, such that the squeezed light is on only during the 'input pulse'. A beam shaper and a telescope create an expanded flat-top intensity profile. The light is then sent to the receiver (memory) consisting of two oppositely oriented ensembles of spin-polarized caesium vapour in paraffin-coated cells and a homodyne detector. The detector signal is processed electronically and used as feedback onto the spins obtained using radiofrequency magnetic field pulses. $\mathbf{b}$, Pulse sequence for the initiation of the memory, storage and verification. Radiofrequency 'feedback' pulses are 0.15 ms long. QND: quantum non-demolition. c, Atomic-level structure illustrating interaction of quantum (dashed lines) and classical (solid lines) modes with the memory.

and by varying the orientation of the squeezed quadrature between $\hat{x}_{\mathrm{L}}$ and $\hat{p}_{\mathrm{L}}$.

The two photonic modes are stored in two ensembles of caesium atoms contained in paraffin-coated glass cells (Fig. 1a) with the ground-state coherence time around $30 \mathrm{~ms}$ (ref. 3). $\omega_{0}$ is bluedetuned by $\Delta=855 \mathrm{MHz}$ from the $F=4 \rightarrow F^{\prime}=5$ of the D2 transition (Fig. 1c). Atoms are placed in a magnetic field that leads to the precession of the ground-state spins with the Larmor frequency $\omega_{\mathrm{L}}=2 \pi \cdot 322 \mathrm{kHz}$. This ensures that the atoms efficiently couple to the entangled $\omega_{ \pm}=\omega_{0} \pm \omega_{\mathrm{L}}$ sidebands of light ${ }^{3}$. The two ensembles 1(2) are optically pumped in $F=4, m_{F}=4(-4)$ states, respectively, which leads to the opposite orientation of their macroscopic spin components $J_{x 1}=-J_{x 2}=J_{x}$.

The atomic memory is conveniently described by two sets $c, s$ of non-local, that is, joint for the two separate memory cells, canonical atomic operators $x_{\mathrm{Ac}}=\left(J_{y 1}{ }^{\mathrm{rot}}-J_{y 2}{ }^{\mathrm{rot}}\right) / \sqrt{2 J_{x}}, p_{\mathrm{Ac}}=\left(J_{z 1}{ }^{\mathrm{rot}}+\right.$ $\left.J_{z 2}{ }^{\text {rot }}\right) / \sqrt{2 J_{x}}, x_{\mathrm{As}}=-\left(J_{z 1}{ }^{\text {rot }}-J_{z 2}{ }^{\text {rot }}\right) / \sqrt{2 J_{x}}, p_{\text {As }}=\left(J_{y 1}{ }^{\text {rot }}+J_{y 2}{ }^{\text {rot }}\right) / \sqrt{2 J_{x}}$, where the superscript rot denotes spin operators in a frame rotating at $\omega_{\mathrm{L}}$. It can be shown that the cosine and sine light modes couple only to the atomic $c$ and $s$ modes, respectively ${ }^{32}$. As a consequence, in the protocol described below the upper (lower) entangled sideband mode of light is stored in the 1 (2) memory cell, respectively. As the equations describing the interaction are the same, we omit the indices $c, s$ from now on.

Light emitted from the sender station to the receiver (memory) station consists of quantum $x$-polarized modes and a strong $y$ polarized part that serves as the driving field for interaction with atoms and as the local oscillator for the subsequent homodyne measurement (Fig. 1). The interaction of light and a gas of
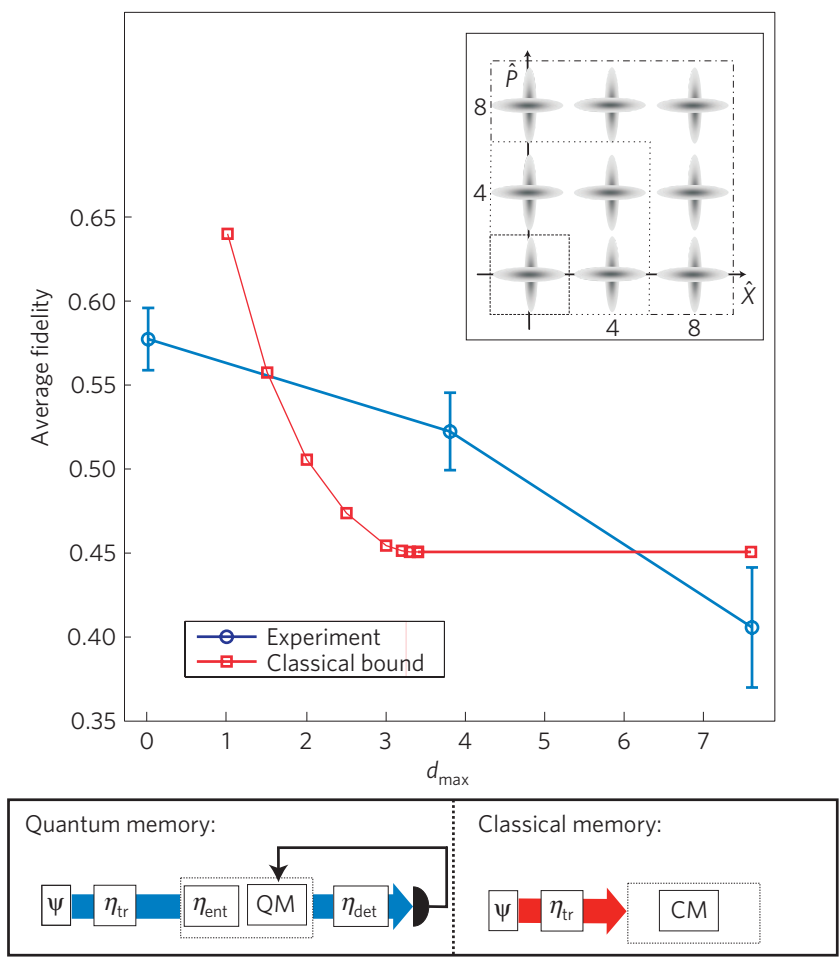

Figure 2 | Fidelities. The graph shows the values of the experimental fidelity (circles) and the theoretical benchmark values (squares) as a function of the size of the set of states $d_{\max }$ with one vacuum unit of displacement corresponding to $d_{\max }=1 / \sqrt{2}$. The inset illustrates the alphabet of states used in the experiment. The three sets of states with $d_{\max }=0 ; 3.8 ; 7.6$ used for the determination of the experimental values of the fidelity plotted in the graph are shown in the inset within dashed, dotted and dashed-dotted squares, respectively. The panel in the bottom shows schematically the propagation channels used in the calculations of the fidelity of the quantum memory (left part) and of the benchmark fidelity of the classical memory (right part). The error bars on the experimental data represent the standard deviations of the results, where all statistical and systematic errors have been included.

spin-polarized atoms under our experimental conditions can be described by the equations ${ }^{32}$ :

$$
\begin{aligned}
& x_{\mathrm{A}}{ }^{\prime}=\sqrt{1-\frac{\kappa^{2}}{Z^{2}}} x_{\mathrm{A}}+\kappa p_{\mathrm{L}}, \quad p_{\mathrm{A}}{ }^{\prime}=\sqrt{1-\frac{\kappa^{2}}{Z^{2}}} p_{\mathrm{A}}-\frac{\kappa}{Z^{2}} x_{\mathrm{L}} \\
& x_{\mathrm{L}}{ }^{\prime}=\sqrt{1-\frac{\kappa^{2}}{Z^{2}}} x_{\mathrm{L}}+\kappa p_{\mathrm{A}}, \quad p_{\mathrm{L}}{ }^{\prime}=\sqrt{1-\frac{\kappa^{2}}{Z^{2}}} p_{\mathrm{L}}-\frac{\kappa}{Z^{2}} x_{\mathrm{A}}
\end{aligned}
$$

where the coupling constant $\kappa$ is a function of light intensity, density of atoms and interaction time, and $Z^{2}=6.4$ is a function of the detuning alone. In the limit $\kappa \rightarrow Z$ these equations describe a swap of operators for light and atoms, that is, a perfect memory followed by squeezing by a factor $Z^{2}$. However, in our experiment the swapping time is too long compared with the atomic decoherence time. To speed up the memory process we add a quantum measurement and feedback steps to this swap operation.

The sequence of operations of the quantum memory protocol is shown in Fig. 1b. We start the memory protocol with initializing the atomic memory state in a spin-squeezed state (SSS). The spin-squeezed state with $\operatorname{Var}\left(x_{\mathrm{A}}\right)=0.43(3)$ and $\operatorname{Var}\left(p_{\mathrm{A}}\right)=1.07(5)$ is generated ${ }^{3,7}$ starting from a (nearly) coherent spin state with $\operatorname{Var}\left(x_{\mathrm{A}}\right)=0.55(4)$ by the sequence (Fig. 1b-preparation of initial state) of the 'quantum non-demolition spin-squeezing pulse' and a 
Table 1 | Initial states of light and stored memory states.

\begin{tabular}{cccccccl}
\multicolumn{7}{c}{ Initial states $\Psi_{\mathbf{i}}$} & \multicolumn{5}{c}{ Stored states } & Overlap \\
\hline$\left\langle x_{\mathrm{L}}\right\rangle$ & $\left\langle p_{\mathrm{L}}\right\rangle$ & $\phi$ & $\left\langle p_{\mathrm{A}}{ }^{\text {fin }}\right\rangle$ & $\left\langle x_{\mathrm{A}}{ }^{\text {fin }}\right\rangle$ & $\operatorname{Var}\left(p_{\mathrm{A}}{ }^{\text {fin }}\right)$ & $\operatorname{Var}\left(x_{\mathrm{A}}{ }^{\text {fin }}\right)$ & $\Theta_{\mathrm{i}}$ \\
\hline 0.0 & 0.0 & 0 & -0.06 & 0.25 & $0.52(2)$ & $1.99(3)$ & 0.62 \\
0.0 & 3.8 & & -0.06 & 3.19 & & & 0.60 \\
3.8 & 0.0 & & -3.47 & -0.42 & & & 0.57 \\
3.8 & 3.8 & & -3.39 & 2.89 & & 0.49 \\
0.0 & 0.0 & 90 & -0.07 & 0.06 & $1.95(6)$ & $0.73(1)$ & 0.55 \\
0.0 & 3.8 & & -0.06 & 3.14 & & & 0.42 \\
3.8 & 0.0 & & -3.22 & 0.48 & & & 0.46 \\
3.8 & 3.8 & & -3.21 & 3.59 & & 0.50 \\
0.0 & 7.6 & 0 & -0.03 & 6.30 & $0.55(2)$ & $2.01(4)$ & 0.49 \\
7.6 & 0.0 & & -6.83 & -0.46 & & 0.37 \\
3.8 & 7.6 & & -3.20 & 6.07 & & & 0.35 \\
7.6 & 3.8 & & -6.54 & 2.80 & & & 0.22 \\
7.6 & 7.6 & & -6.40 & 6.03 & & & 0.15 \\
0.0 & 7.6 & 90 & -0.08 & 6.24 & $2.12(8)$ & $0.78(3)$ & 0.18 \\
7.6 & 0.0 & & -6.37 & 0.59 & & & 0.35 \\
3.8 & 7.6 & & -3.13 & 6.75 & & & 0.32 \\
7.6 & 3.8 & -6.38 & 3.79 & & & 0.43 \\
7.6 & 7.6 & -6.36 & 6.72 & & & 0.27 \\
\hline
\end{tabular}

The three first columns show the mean displacements and the squeezing phase $(\phi=0$ corresponds to $x_{L}$ being squeezed) of the initial pure light states. The next four columns show the mean values and variances of the atomic states after the storage. The last column shows the overlap between the initial pure light states and the stored atomic states. Vacuum-state variances are 0.5 . The uncertainties on the variances are calculated as the standard deviation of the variances within each subgroup of the input states.

feedback into both the $c$ and $s$ modes. The feedback is achieved with pulses of the magnetic field at the frequency $\omega_{\mathrm{L}}$ applied to the two cells. The memory input light pulse is then sent, followed by the measurement of the output light operator $x_{\mathrm{L}}^{\prime}$ by the polarization homodyne detection. The measurement result is fed back onto the $p_{\mathrm{A}}{ }^{\prime}$ with a gain $g$. The resulting $x_{\mathrm{A}}{ }^{\text {fin }}$ and $p_{\mathrm{A}}{ }^{\text {fin }}$ for the optimized $g$ and $\kappa=1$ can be found from equation (1)

$$
x_{\mathrm{A}}{ }^{\text {fin }}=\sqrt{1-\frac{1}{Z^{2}}} x_{\mathrm{A}}+p_{\mathrm{L}} \text { and } \quad p_{\mathrm{A}}{ }^{\mathrm{fin}}=-x_{\mathrm{L}}
$$

In the absence of decoherence the operator $x_{\mathrm{L}}$ is perfectly mapped on the memory operator $p_{\mathrm{A}}{ }^{\text {fin }}$. The operator $p_{\mathrm{L}}$ is stored in $x_{\mathrm{A}}{ }^{\text {fin }}$ with the correct mean value $\left\langle p_{\mathrm{L}}\right\rangle=\left\langle x_{\mathrm{A}}{ }^{\text {fin }}\right\rangle$ (as $\left.\left\langle x_{\mathrm{A}}\right\rangle=0\right)$ but with an additional noise due to $x_{\mathrm{A}}$.

The ability to reproduce the correct mean values of the input state in the memory by adjusting $g$ and $\kappa$ is a characteristic feature of our protocol that arguably makes it better suited for storage of multiphoton states compared with, for example, electromagnetically induced transparency and photon-echo approaches where such an adjustment, to the best of our knowledge, has not been demonstrated.

The deleterious noise of the initial state of atoms $x_{\mathrm{A}}$ is suppressed by the initial spin-squeezing sequence and by the extra factor $\sqrt{1-1 / Z^{2}}=0.92$ due to the swap interaction. In the absence of passive (reflection) losses for light and atomic decoherence we find from equation (2) the expected fidelity of 0.95 and 0.61 for the states squeezed with the $\phi=0^{\circ}$ and $\phi=90^{\circ}$, respectively, with the mean fidelity of 0.78 .

The true quantum character of our memory is preserved despite substantial transmission losses schematically shown in the bottom of Fig. 2. Entangled states of light are sent to the receiver memory station through a channel with the transmission coefficient $\eta_{\text {tr }}=0.80(4)$ (which includes the OPA output coupling efficiency 0.97 ) resulting in the 'memory input state', $\rho_{\text {in }}$, with $\operatorname{Var}\left(x_{\mathrm{L}} \cdot \cos (\phi)-p_{\mathrm{L}} \cdot \sin (\phi)\right)=0.20(2)$ and $\operatorname{Var}\left(x_{\mathrm{L}} \cdot \sin (\phi)+p_{\mathrm{L}} \cdot \cos (\phi)\right)=1.68(9)$. The entrance (reflection) losses at the windows of the memory cells lead to further attenuation by the factor $\eta_{\text {ent }}=0.90(1)$. Between the interaction and detection light experiences losses described by the detection efficiency $\eta_{\text {det }}=0.79(2)$ (see the Methods section).

Following the storage time of $1 \mathrm{~ms}$ between the end of the input pulse and the beginning of the verifying pulse (see Fig. 1b for the time sequence) we measure the atomic operators with a verifying probe pulse. The measured mean values and variances of the atomic operators $x_{\mathrm{A}}{ }^{\text {fin }}$ and $p_{\mathrm{A}}{ }^{\text {fin }}$ are summarized in Table 1 (see the Methods section for calibration of the atomic operators). From these values and the loss parameters, we can calculate the noise added during the storage process that comes on top of the noise added by transmission and entrance losses. We find that the memory adds 0.47 (6) to $\operatorname{Var}\left(x_{\mathrm{A}}{ }^{\text {fin }}\right)$ and 0.38 (11) to $\operatorname{Var}\left(p_{\mathrm{A}}{ }^{\text {fin }}\right)$, whereas for the ideal memory, according to equation (2), we expect the additional noise to be 0.36 (5) (due to the finite squeezing of the initial atomic operator $x_{\mathrm{A}}$ ) and 0 , for the two quadratures, respectively. This added noise can be due to atomic decoherence, uncancelled noise from the initial antisqueezed $p_{\mathrm{A}}$ quadrature and technical noise from the EOMs.

The overlap integrals between the stored states and the initial pure states are given in the Table 1. The average fidelities calculated from the overlap values for square input distributions with the size $d_{\max }=0,3.8$ and 7.6 are plotted in Fig. 2. The choice of the interaction strength $\kappa=1$ minimizes the added noise but leads to the mismatch between the mean values of the stored atomic state and of the initial pure state of light by the factor $\sqrt{\eta_{\mathrm{ent}} \cdot \eta_{\mathrm{tr}}}=0.85$. This mismatch is the reason for the reduction of the experimental fidelity for states with larger displacements.

The classical benchmark memory fidelity is calculated ${ }^{28}$ from the overlap of displaced $\Psi_{\mathrm{i}}$ states with the states stored in the (hypothetical) classical memory positioned in place of the quantum memory (see the panel in the bottom of Fig. 2). This ensures that the classical memory has the same input state $\rho_{\text {in }}$ as the quantum memory that is $\Psi_{\mathrm{i}}$ propagated through the transmission channel with $\eta_{t r}=0.80$. The benchmark fidelity is found as an average overlap for an input distribution within a square $\left\{\left|\left\langle x_{\mathrm{L}}\right\rangle\right|,\left|\left\langle p_{\mathrm{L}}\right\rangle\right| \leq d_{\max }\right\}$ with all input states with the mean values within the square having equal probability. The squeezing of the pure input states is fixed to the experimental value of $6 \mathrm{~dB}$, and all phases of squeezing are allowed. The upper bound values on the classical benchmark fidelity are plotted in Fig. 2. The benchmark values have been obtained by first truncating the Hilbert space to a finite photon number and then solving the finite-dimensional optimization employing semi-definite programming. The result of the truncation of the Hilbert space is the constant upper bound of 0.45 of the benchmark for $d_{\max }>3.5$ (see Supplementary Information), whereas the actual benchmark decreases further for a larger $d_{\max }$ (the benchmark for an infinite Gaussian distribution ${ }^{28}$ of displacements is 0.38 ).

For the experimental alphabet with $d_{\max }=3.8$ the experimental fidelity (the average overlap integral for the top eight representative states in Table 1) is higher than the classical benchmark value, which proves the true quantum nature of the memory.

Outperforming the classical benchmark means that our memory is capable of preserving entanglement in the case when one of the two entangled modes is stored whereas the other is left propagating, which is the case, for example, in the quantum repeater. Using experimentally obtained values of the added noise we evaluate the performance of our memory for the protocol where the upper sideband $\hat{a}_{+}$mode is stored in one of the memory cells whereas the other entangled mode $\hat{a}_{-}$is left as a propagating light mode. We find the Einstein-Podolsky-Rosen variance between the stored mode and the propagating mode to be $1.52(-1.2 \mathrm{~dB}$ below the 
separability criterion), which corresponds to the lower bound on the entanglement of formation of $\sim 1 / 7$ ebit (see Supplementary Information for details of the calculation). This version of the memory can be implemented by splitting the $\hat{a}_{-}$and $\hat{a}_{+}$modes, which can be accomplished by a narrow-band optical cavity.

We have experimentally demonstrated the deterministic quantum memory for continuous-variable multiphoton entangled states. The present memory lifetime is limited by collisions and residual magnetic fields leading to the decoherence time of $30 \mathrm{~ms}$. The fidelity can be further improved by means of reduction of the reflection losses, increasing the initial atomic spin squeezing and reduction of the atomic decoherence. Our approach is feasible also for non-Gaussian continuous-variable multiphoton states. Stored states can be directly processed in the atomic memory, as in the repeater scheme ${ }^{18}$, measured, as in multiround protocols ${ }^{22}$, or transferred onto another processor by means of teleportation ${ }^{3}$.

\section{Methods}

Verification. Atomic memory state tomography is carried out by measuring the $x_{\mathrm{L}}{ }^{\prime}$ of the verification pulse equation (1). We run a series of measurements of $p_{\mathrm{A}}{ }^{\text {fin }}$ and $x_{\mathrm{A}}{ }^{\text {fin }}$, the latter carried out after applying a magnetic $\pi$-pulse before the verification pulse, for many copies of the same input state of light. Owing to the Gaussian statistics of the states, the mean values and the variances of $x_{\mathrm{A}}{ }^{\text {fin }}$ and $p_{\mathrm{A}}{ }^{\text {fin }}$ are sufficient for a complete description of the atomic state.

Calibrations. Before carrying out the storage, we calibrate the interaction strength $\kappa$ and the feedback gain $g$, such that the mean values of the light state inside the memory (that is, after the entrance loss) are transferred faithfully. $\kappa$ is calibrated by creating a mean value $\left\langle p_{\mathrm{L}}{ }^{\text {input }}\right\rangle$ in the input pulse. The mean is stored in the atomic $x_{\mathrm{A}}{ }^{\prime}$, which is read out after the magnetic $\pi$-pulse with the probe pulse. The measured mean of the probe pulse is then $\left\langle x_{\mathrm{L}}{ }^{\text {probe }}\right\rangle=\kappa^{2}\left\langle p_{\mathrm{L}}{ }^{\text {input }}\right\rangle$, from which $\kappa^{2}$ is determined. Using similar methods we can calibrate the electronic feedback gain $g$.

Generation of the displaced squeezed input states. The OPA, which is pumped by the second harmonic of the master laser and generates the entangled squeezed vacuum states, is seeded with a few microwatts of the master laser light with the carrier frequency $\omega_{0}$, which is amplitude and phase modulated by two EOMs at a frequency of $322 \mathrm{kHz}$, thus creating coherent states in the $\pm 322 \mathrm{kHz}$ sidebands around $\omega_{0}$ (Fig. 1). With such a modulated seed, the output of the OPA is a displaced two-mode squeezed state. The output of the OPA is mixed at a polarizing beam splitter with the strong local oscillator (driving) field from the master laser.

Losses. To calculate the mean values and variances of the stored state and the input states, we need to know the optical losses. The total losses $\eta_{\text {tot }}$ can be divided into three parts, the channel propagation transmission $\eta_{\mathrm{tr}}$ from the OPA to the front of the memory cells (including the OPA output efficiency 0.97), the entrance transmission $\eta_{\text {ent }}$ and the detection efficiency $\eta_{\text {det }}$ such that $\eta_{\text {tot }}=\eta_{\text {tr }} \cdot \eta_{\text {ent }} \cdot \eta_{\text {det }}$ (all of the $\eta$ terms are intensity transmission coefficients). From the measurement of the quadratures of the squeezed light (with variances $0.29(1)$ and $1.34(6)$ ), we find the total losses $\eta_{\text {tot }}=0.567(35)$. We measure the transmission through the cells of $0.817(20)$, the transmission through the optics after the cells of 0.889 (10) and estimate the efficiency of the photodiodes to be 0.98 (1). Assigning one-half of the losses through the cells to the entrance losses and the other half to the detection losses we find $\eta_{\text {ent }}=\sqrt{0.817}=0.90(1), \eta_{\text {det }}=\sqrt{0.817} \cdot 0.889 \cdot 0.98=0.79(2)$ and $\eta_{\mathrm{tr}}=\eta_{\mathrm{tot}} /\left(\eta_{\text {ent }} \eta_{\mathrm{det}}\right)=0.80(4)$

Received 4 April 2010; accepted 13 September 2010; published online 7 November 2010

\section{References}

1. Kimble, H. J. The quantum internet. Nature 453, 1023-1030 (2008).

2. Lvovsky, A. I., Sanders, B. C. \& Tittel, W. Optical quantum memory. Nature Photon. 3, 706-714 (2009).

3. Hammerer, K., Sørensen, A. S. \& Polzik, E. S. Quantum interface between light and atomic ensembles. Rev. Mod. Phys. 82, 1041-1093 (2010).

4. Ralph, T. C. \& Lam, P. K. A bright future for quantum communications. Nature Photon. 3, 671-673 (2009).

5. Furusawa, A. \& Takei, N. Quantum teleportation for continuous variables and related quantum information processing. Phys. Rep. 443, 97-119 (2006)
6. Reid, M. D. et al. The Einstein-Podolsky-Rosen paradox: From concepts to applications. Rev. Mod. Phys. 81, 1727-1751 (2009).

7. Sherson, J., Julsgaard, B. \& Polzik, E. S. Deterministic atom-light quantum interface. Adv. At. Mol. Opt. Phys. 54, 81-130 (2007).

8. Furusawa, A. et al. Unconditional quantum teleportation. Science 282, 706-709 (1998).

9. Sherson, J. F. et al. Quantum teleportation between light and matter. Nature 443, 557-560 (2006).

10. Nunn, J. et al. Multimode memories in atomic ensembles. Phys. Rev. Lett. 101, 260502 (2008).

11. Hosseini, M. et al. Coherent optical pulse sequencer for quantum applications. Nature 461, 241-245 (2009).

12. Grosshans, F. et al. Quantum key distribution using Gaussian-modulated coherent states. Nature 421, 238-241 (2003).

13. Ourjoumtsev, A., Jeong, H., Tualle-Brouri, R. \& Grangier, P. Generation of optical 'Schrödinger cats' from photon number states. Nature $\mathbf{4 4 8}$ 784-786 (2007)

14. Takahashi, H. et al. Entanglement distillation from Gaussian input states. Nature Photon. 4, 178-181 (2010).

15. Dong, R. et al. Experimental entanglement distillation of mesoscopic quantum states. Nature Phys. 4, 919-923 (2008).

16. Gottesman, D., Kitaev, A. \& Preskill, J. Encoding a qubit in an oscillator. Phys. Rev. A 64, 012310 (2001).

17. Lund, A. P., Ralph, T. C. \& Haselgrove, H. L. Fault-tolerant linear optical quantum computing with small-amplitude coherent states. Phys. Rev. Lett. 100, 030503 (2008)

18. Brask, J. B., Rigas, I., Polzik, E. S., Andersen, U. L. \& Sørensen, A. S. Hybrid long-distance entanglement distribution protocol. Phys. Rev. Lett. 105, 160501 (2010).

19. Sangouard, N. et al. Quantum repeaters with entangled coherent states. Preprint at http://arxiv.org/abs/0912.3871 (2010).

20. Browne, D. E., Eisert, J., Scheel, S. \& Plenio, M. B. Driving non-Gaussian to Gaussian states with linear optics. Phys. Rev. A 67, 062320 (2003).

21. Gu, M., Weedbrook, C., Menicucci, N. C., Ralph, T. C. \& van Loock, P. Quantum computing with continuous.variable clusters. Phys. Rev. A 79, 062318 (2009).

22. Lamoureux, L. P., Brainis, E., Amans, D., Barrett, J. \& Massar, S. Provably secure experimental quantum bit-string generation. Phys. Rev. Lett. 94, 050503 (2005).

23. Lloyd, S. Enhanced sensitivity of photodetection via quantum illumination. Science 321, 1463-1465 (2008).

24. Marino, A. M., Pooser, R. C., Boyer, V. \& Lett, P. D. Tunable delay of Einstein-Podolsky-Rosen entanglement. Nature 457, 859-862 (2009).

25. Appel, J., Figueroa, E., Korystov, D., Lobino, M. \& Lvovsky, A. I. Quantum memory for squeezed light. Phys. Rev. Lett. 100, 093602 (2008).

26. Honda, K. et al. Storage and retrieval of a squeezed vacuum. Phys. Rev. Lett. 100, 093601 (2008)

27. Adesso, G. \& Chiribella, G. Quantum benchmark for teleportation and storage of squeezed states. Phys. Rev. Lett. 100, 170503 (2008).

28. Owari, M., Plenio, M. B., Polzik, E. S., Serafini, A. \& Wolf, M. M. Squeezing the limit: Quantum benchmarks for the teleportation and storage of squeezed states. New J. Phys. 10, 113014 (2008).

29. Calsamiglia, J., Aspachs, M., Munoz-Tapia, R. \& Bagan, E. Phys. Rev. A 79, 050301 (2009).

30. Duan, L., Giedke, G., Cirac, J. \& Zoller, P. Inseparability criterion for continuous variable systems. Phys. Rev .Lett. 84, 2722-2725 (2000).

31. Schori, C., Sørensen, J. L. \& Polzik, E. S. Narrowband frequency tunable source of entangled light. Phys. Rev. A 66, 033802 (2002).

32. Wasilewski, W. et al. Single mode quadrature entangled light from room temperature atomic vapour. Opt. Express 17, 14444-14457 (2009).

\section{Acknowledgements}

This work was supported by EU projects QESSENCE, HIDEAS, CORNER, COMPAS, EMALI and COQUIT.

\section{Author contributions}

Experimental group: K.J., W.W., H.K., T.F., B.M.N. and E.S.P. Calculation of the classical benchmark: M.O., M.B.P., A.S. and M.M.W.

\section{Additional information}

The authors declare no competing financial interests. Supplementary information accompanies this paper on www.nature.com/naturephysics. Reprints and permissions information is available online at http://npg.nature.com/reprintsandpermissions. Correspondence and requests for materials should be addressed to E.S.P. 